\title{
Applying the Method for Evaluation of Motivational Aspects on Karuchā Ships Invaders Educational Game with Brazilian Students of Japanese
}

\author{
Juvane Nunes Marciano, Erica Esteves Cunha de Miranda, \\ Leonardo Cunha de Miranda \\ Department of Informatics and Applied Mathematics, \\ Federal University of Rio Grande do Norte (UFRN), Natal, Brazil
}

juvane@ppgsc.ufrn.br, erica@dimap.ufrn.br, leonardo@dimap.ufrn.br

\begin{abstract}
The increasing use of educational games in several learning contexts is noticed, and to reinforce this growth, it is very important to evaluate these tools in several of their aspects. An important aspect is the influence this kind of tool exercises in the learning process. This paper presents the evaluation of motivational aspects of Karuchā Ships Invaders educational game, through a method developed earlier by the authors, demonstrating that these aspects influence positively on the user's will to continue the learning and practice of Japanese language. The study was applied with Brazilian students of Japanese, and the results show that Karuchā Ships Invaders is a good educational tool for Japanese language learning.
\end{abstract}

\section{Introduction}

CALL (Computer Assisted Language Learning) tools are increasingly present in our daily life. There are several kinds of tools that can be classified as CALL - e.g. websites, desktop software and mobile apps - and for each of these kinds, there are several teaching approaches. It is possible to find tutor systems, exercises collections, cooperative online learning, and also games. CALL games are very interesting and engaging for most users, since it blends learning with entertainment and fun. The present work focuses on CALL games and their influence on the motivation and learning of the students, including the development and expansion of evaluation methods to obtain this kind of data. The evaluation of motivational aspects of educational games is important to give the developers several clues about the end-users' approval or disapproval for the game features, and guide the course of development.

This work is placed in the context of a research project whose theme is Japanese language learning supported by computational tools. Therefore, this paper presents new results of our research on this subject. In previous works, we presented the state of the art [Marciano et al. 2012], and the state of the practice of web [Marciano et al. 2013a] and mobile (Android) [Marciano et al. 2013b] platforms of CALL tools for Japanese language. Also, we developed a educational game called Karuchā Ships Invaders [Marciano et al. 2013c] focused on Japanese language learning, and a method of evaluation of motivational aspects of educational media and technology [Marciano et al. 2014]. 
Karuchā Ships Invaders [Marciano et al. 2013c] is a CALL game ${ }^{1}$, in a space invaders' style, focused on Japanese language, developed by our research team in Lua programming language with $\mathrm{MOAI}^{2}$ game engine platform. With it, the student-player can learn and practice knowledge about the most basic Japanese alphabet - i.e. hiragana ${ }^{3}$ - and cultural words related to Japanese culture. The game tells a story where Japanese ships are heading to Brazil and need help to land. To help them, the player has to know the basics of the Japanese language, to pass the commands to the ships. The game offers three basic difficulty modes, i.e. easy, normal and hard, each one increasing velocity and frequency to the ships' fall. Also, it exhibits stories to present the content to the players this is one of the clear educational aspects of Karuchā.

In this work, we present the application of the method developed earlier by the authors [Marciano et al., 2014], on Karuchā Ships Invaders educational game and its results regarding the motivational power of our game in making Japanese language students want to keep their learning. The experiment was accomplished with Brazilian prospective users and provided quantitative and qualitative data, whose analysis will also be detailed in the present work.

This paper is organized as follows: Section 2 introduces the research and methodology scenario; Section 3 presents the results for the evaluation; Section 4 presents the discussion; and Section 5 concludes the paper.

\section{Research and Methodology Scenario}

The method is a heuristic-based method for evaluation of motivational aspects of educational media and technology. The heuristics of the method were developed based on the ARCS Model [Keller, 1987], i.e., divided into four axes: Attention, Relevance, Confidence and Satisfaction. ARCS Model was first developed to be used in traditional courses in Educational, but there are works that used it as a basis to accomplish evaluations with digital educational programs, as in Shellnut et al. (1999), Wongwiwatthananukit (2000) and Huang et al. (2004).

The experiment was divided in six stages: definition, planning, data gathering, calculation, presentation and data analysis and interpretation. Follows the experiment guide for the Data Gathering stage (when the users do participate), which was divided in five steps, to detail and clarify the process:

1. There is a quick presentation of the environment, in which the user is aware where to stay during the experience, what will be the steps of the experiment, know about the cameras and fill a Free and Clarified Consent Form, in order to allow the experimenters to collect the data.

2. The experimenter follows a presentation guide (whose accomplishment should take up to 10 minutes):

\footnotetext{
${ }^{1}$ Available for free download at http://www.karucha.pairg.dimap.ufrn.br.

${ }^{2}$ http://www.getmoai.com.

${ }^{3}$ Hiragana is the most basic Japanese alphabet, used for native words for which there are no kanji, or an unusual kanji, and also for grammatical particles, suffixes, verb and adjective inflections. Hiragana is also used to show the pronunciation of kanji as a reading aid, like a subtitle, what is called furigana.
} 
a. Explain about Karuchā (download, plot, educational features and rules);

b. Explain about the motivational testing (where the user's ability or Japanese language level are not being evaluated/judged, but the main goal is to determine the motivational power of the tool);

c. Present the game menu, options and keyboard shortcuts;

d. Present the gameplay, playing a level as an example.

3. The user is free to explore the game for up to 40 minutes (it is also explained that if the user desires to stop using the tool, they are able to do that at any moment). In this step, the experimenter note observations about the user-tool interaction.

4. The user answers the motivational questionnaire, being aware that they can ask for help due to possible doubts.

5. The experimenter mediates the final interview with the user, based on the predefined questions, but being able to adapt the flow accordingly to observations about the user during the usage of the game.

For this evaluation, we needed users who were involved somehow with Japanese language and culture. We searched between students and also professors; and, since it is a game we are evaluating, we searched for the users among people who were already computer game players and people that are not (searching for a player and a non-player for each of the profiles). The user profiles we searched for to accomplish the evaluation were: i) aspirants to the study of Japanese language (no previous knowledge); ii) beginners in the study of Japanese language (some knowledge in kanas ${ }^{4}$ ); iii) intermediate students of Japanese language (JLPT ${ }^{5} \mathrm{~N} 5$ or some kanji ${ }^{6}$ ); iv) advanced students of Japanese language (JLPT N4 or N3); and v) expert students of Japanese language (JLPT $\mathrm{N} 2$ or N1). These profiles were chosen because we would like to observe if the game is suitable for all these levels of knowledge, and also to see whether motivation varies due to the knowledge when playing the game.

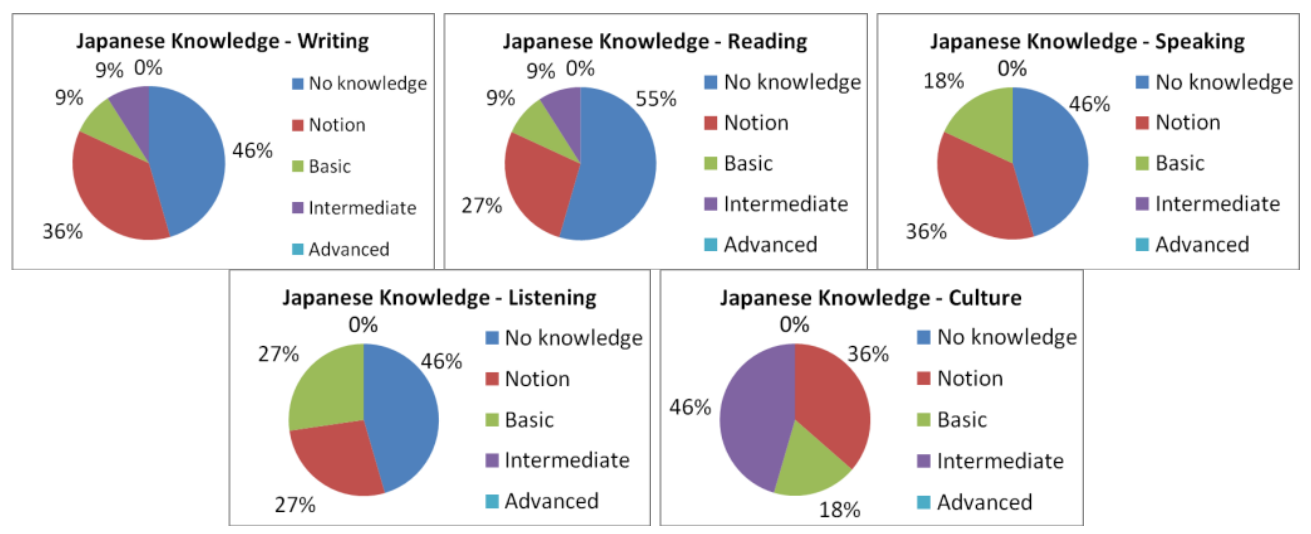

Fig. 1. Diagrams that detail user characteristics.

\footnotetext{
${ }^{4}$ Kanas are the two basic alphabets of Japanese language, i.e. hiragana and katakana, which compose the former reading and writing studies of the language.

${ }^{5}$ JLPT is the Japanese Language Proficiency Test, divided in five levels, in which N5 is the easiest and $\mathrm{N} 1$ is the most difficult level.

${ }^{6}$ Kanji are ideogram characters of the Japanese language, more complicated than kana, and much more numerous (each kana alphabet has 48 basic symbols, whilst kanji are 2,136 of most usage (taught mandatorily), but the total amount is not exact.
} 
We were able to accomplish the evaluation with 11 users, one at a time. The detailed profile of the users is described in the diagrams (Fig. 1), which detail the following information: Japanese knowledge of the users in the four language skills - i.e. writing, reading, speaking and listening - and Japanese cultural knowledge. From all the 11 users, the age varies from 16 to 23 years old; $73 \%$ are male and $23 \%$ are female; and $91 \%$ declared themselves as computer game players, against $9 \%$ non-players.

\section{Results}

\subsection{Quantitative Results}

The quantitative results will be presented in tables and figures with diagrams, divided into Log Flow and Results of Severity Analysis of the Motivational Questionnaire.

Log Flow. The following figures represent the flow of the users through the game, recorded in the log file. Fig. 2 shows the maximum level each user achieved, in each one of the three most basic difficulty modes, i.e. easy, medium and hard ${ }^{7}$. Fig. 3 shows a flow of a specific user (U3) through levels of the hard mode, showing how many times he played, failed and won each level and the maximum level reached. Fig. 4 is a counter of how many times each user used the context-help ${ }^{8}$ in each level.

An interesting thing to analyze about Fig. 2 is that few users played all the difficulty modes, and crossing data with the profile, the users who played in hard mode were the ones with more knowledge about Japanese language. Another relevant observation is that a user (U9) with no knowledge about the idiom was able to achieve a high level (level 19) in easy mode, and crossing data with Fig. 4, it is possible to infer that the context-help feature was very important for this accomplishment. U8 and U10 also fit in this description. The U1, instead, took another approach: crossing the diagram with observation data, it was possible to see that this user was trying to learn the symbols through several repetitions, and that is why, even taking all the given time, he only reached level 3.

Fig. 3 shows a representation of a game flow through hard mode of a specific user (U3). It is interesting to notice that the levels he failed the most had bosses (level 9 and 12), but the previous boss levels ( 3 and 6) were won in first try. This shows that the game difficulty is increasing with the levels. Also, it is perceived that the level he was not able to pass was an only-boss level (level 15). Even if the user is an intermediate Japanese student, the game was able to provide a challenge, showing that in fact it suits since beginners until advanced Japanese students.

About Fig. 4, the main inference is the relevance of the context-help feature. Only three of the users did not access the feature, being two of them intermediate/advanced Japanese students. The number of times this feature was used is noticeable, in a period of

\footnotetext{
${ }^{7}$ Each difficulty mode has 30 levels, in which every three levels there are bosses mixed with hiragana, and the levels 15 and 30 are only-boss levels.

${ }^{8}$ Context-help is a feature with educational purposes, in which the student-player can consult, during the level, the reading of the kanas or bosses. It is available in easy mode for all the kanas and bosses presented so far, and in medium mode, only for the kanas and bosses of the present level.
} 
40 minutes, there were users able to access it for about 150 times. Except for the ones who did not used context-help, the minimum of times a user accessed it during the experiment was about 30 times. This feature was not originally in the initial concept of Karuchā, but the importance of its addition to the experiment is highly proved by this diagram, and also by the logs, observations and users' answers.

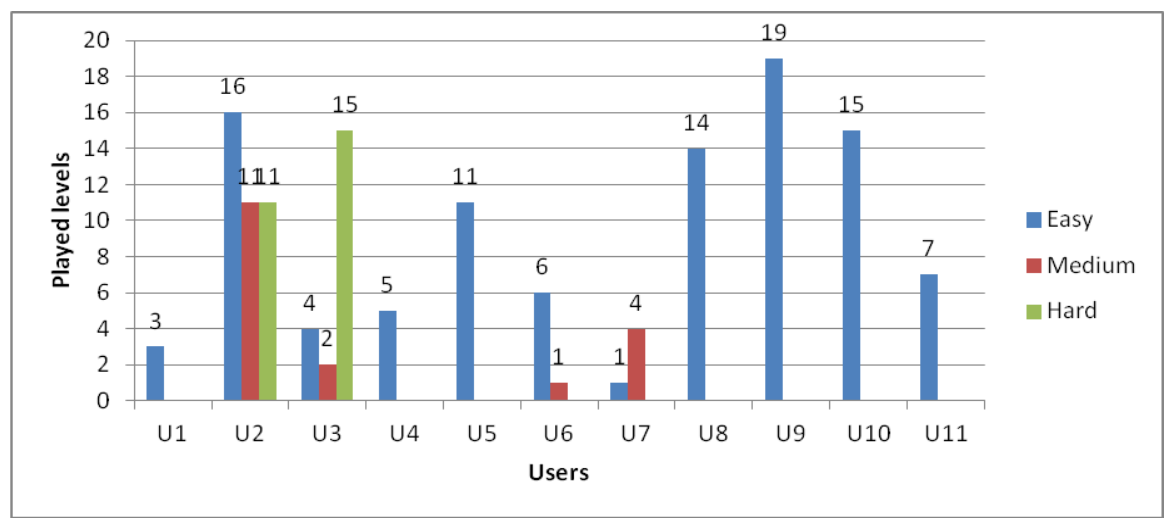

Fig. 2. Maximum level the users achieved.

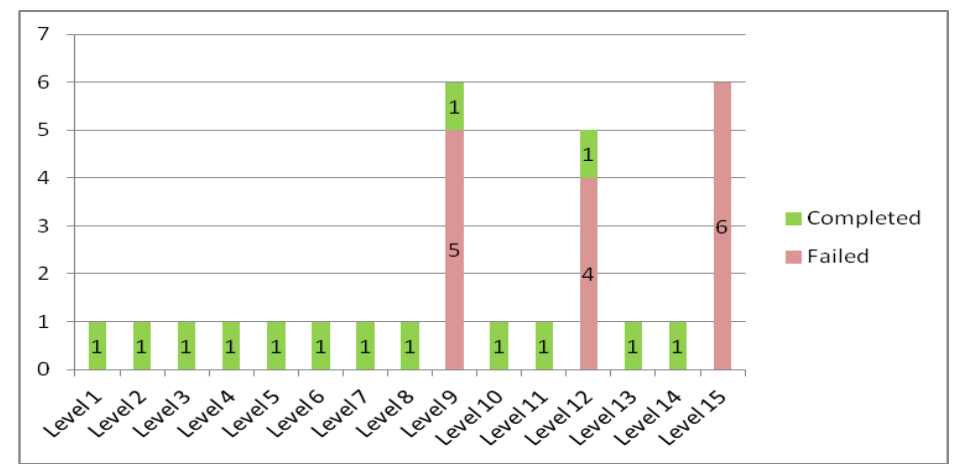

Fig. 3. A flow through levels of hard mode, from user "U3".

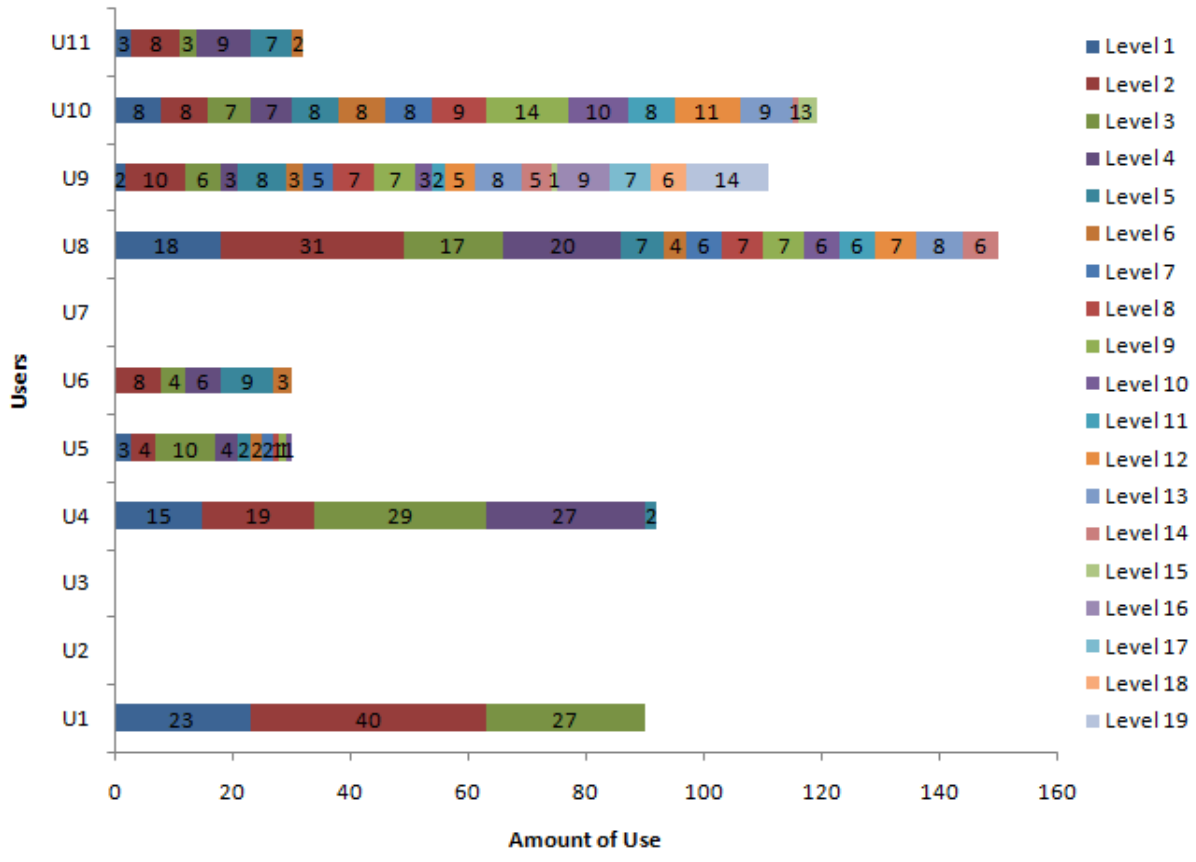

Fig. 4. Counter of use of the context-help in each level. 
Results of Severity Analysis. Fig. 5 is a table containing the evaluation of severity analysis for the motivational questionnaire, where there is represented an average of each question's response including all the users' responses. The first group is for Attention, second for Relevance, third from Confidence and fourth from Satisfaction. It is noticed that the lower scores were related to Confidence and Satisfaction, and the analysis of what may have caused it is presented on qualitative results. Still, crossing profile data with the answers it is perceived that lower scores were given by advanced Japanese students or regular computer game players, which are more rigorous users in this context. Their answers were very important and should be well analyzed in order to improve the motivational aspects of Karuchā educational game.

\begin{tabular}{|c|ccccccccccccc|c|c|c|}
\hline Question & U1 & U2 & U3 & U4 & U5 & U6 & U7 & U8 & U9 & U10 & U11 & Min & Max & Mode & Average \\
\hline 1 & 5 & 5 & 4 & 4 & 5 & 4 & 5 & 4 & 5 & 4 & 5 & 4 & 5 & 5 & 4,55 \\
2 & 5 & 5 & 3 & 4 & 5 & 3 & 4 & 4 & 4 & 3 & 4 & 3 & 5 & 4 & 4,00 \\
3 & 5 & 4 & 4 & 4 & 4 & 5 & 4 & 5 & 4 & 4 & 4 & 4 & 5 & 4 & 4,27 \\
4 & 4 & 5 & 5 & 5 & 5 & 5 & 5 & 5 & 5 & 5 & 5 & 4 & 5 & 5 & 4,91 \\
5 & 4 & 3 & 5 & 3 & 4 & 5 & 3 & 3 & 5 & 5 & 4 & 3 & 5 & 3 & 4,00 \\
6 & 4 & 5 & 3 & 3 & 5 & 5 & 3 & 3 & 3 & 3 & 3 & 3 & 5 & 3 & 3,64 \\
7 & 4 & 4 & 3 & 4 & 3 & 4 & 4 & 5 & 5 & 3 & 3 & 3 & 5 & 4 & 3,82 \\
\hline 8 & 5 & 4 & 3 & 5 & 5 & 5 & 2 & 3 & 5 & 5 & 3 & 2 & 5 & 5 & 4,09 \\
9 & 4 & 4 & 2 & 4 & 5 & 5 & 2 & 5 & 5 & 5 & 4 & 2 & 5 & 5 & 4,09 \\
10 & 5 & 5 & 2 & 4 & 4 & 5 & 3 & 5 & 5 & 5 & 4 & 2 & 5 & 5 & 4,27 \\
11 & 5 & 4 & 4 & 5 & 4 & 3 & 3 & 3 & 4 & 4 & 2 & 2 & 5 & 4 & 3,73 \\
12 & 4 & 4 & 4 & 4 & 4 & 5 & 4 & 4 & 4 & 4 & 2 & 2 & 5 & 4 & 3,91 \\
13 & 5 & 4 & 5 & 5 & 5 & 5 & 5 & 4 & 4 & 4 & 4 & 4 & 5 & 5 & 4,55 \\
\hline 14 & 5 & 5 & 5 & 4 & 5 & 5 & 3 & 3 & 5 & 5 & 5 & 3 & 5 & 5 & 4,55 \\
15 & 3 & 5 & 1 & 3 & 4 & 3 & 4 & 3 & 2 & 3 & 2 & 1 & 5 & 3 & 3,00 \\
16 & 5 & 5 & 1 & 3 & 5 & 5 & 3 & 3 & 3 & 3 & 5 & 1 & 5 & 5 & 3,73 \\
17 & 4 & 3 & 4 & 3 & 4 & 5 & 4 & 3 & 4 & 3 & 5 & 3 & 5 & 4 & 3,82 \\
\hline 18 & 4 & 4 & 1 & 4 & 5 & 5 & 3 & 3 & 4 & 4 & 4 & 1 & 5 & 4 & 3,73 \\
\hline
\end{tabular}

Fig. 5. Severity table of the motivational questionnaire's answers.

\subsection{Qualitative Results}

The qualitative results of this experiment were extracted from observations during the usage step (step 3), comments written spontaneously by the users in the motivational questionnaire (step 4) and interview answers (step 5). Through this data, it was possible to confirm some issues the developers were already aware because of the interface evaluation, that were not fixed until the experiment. For example, issues with size and choice of some bosses' pictures, commented by some of the participants. Also, some animation effects were discovered as giving the wrong message, such as the abduction animation, that could pass the idea of destruction of the ships, instead of the intended help to land. A positive fact detected about the design is that several of the users demonstrated more interest for the game after seeing its first graphics, as the menu screen and a level screen.

Other issue detected through users' suggestions was in the context-help feature; this feature's importance and relevance was already confirmed in the quantitative data analysis, but the users gave ideas to improve it. One of the ideas is to offer help of all levels until the current level, being available to navigate through levels' new kanas. Also, it was suggested a boss context-help, following the same format of kana context-help. Issues related to statistics presented at the end of each level were also detected. It was suggested that the stats presented not only the missed kanas, but also their reading in roma-ji, i.e., the roman letters. 
Some users also commented they would like to compare their results with other student-players, in order to get extra motivation to improve their skills in the game. Another interesting feature suggested was to the game give recommendations to the player, depending on how the level was finished. For example, if the player won the level, but took too many hits, the game could give a message like "You may need to repeat this level to improve your learning". Other suggestions embraced the development of an elaborated scoring system, to be used for comparison/competition between users; the development of online server and features, such as achievements, timeline stats, social share of results; and also, portability to other OS, such as Linux and Mac. All these punctual repairs contribute strongly to improve the motivational power of Karuchā, since every comment that is analyzed and treated properly makes users more comfortable in using the game, and the flow becomes more pleasant.

The following figures show some user reactions during the accomplishment of the evaluation. While playing the game, interaction with the game was registered (Fig. 6a), and also users' facial expressions (Fig. 6b). It is noticed that various users had good moments during the usage of the tool, which is mainly perceived in Fig. 6, through visual expressions $^{9}$ of joy. Also, there were moments of confusion, doubts and frustration, which were caused sometimes for interface issues, and sometimes for the game itself, where the player can win or lose.

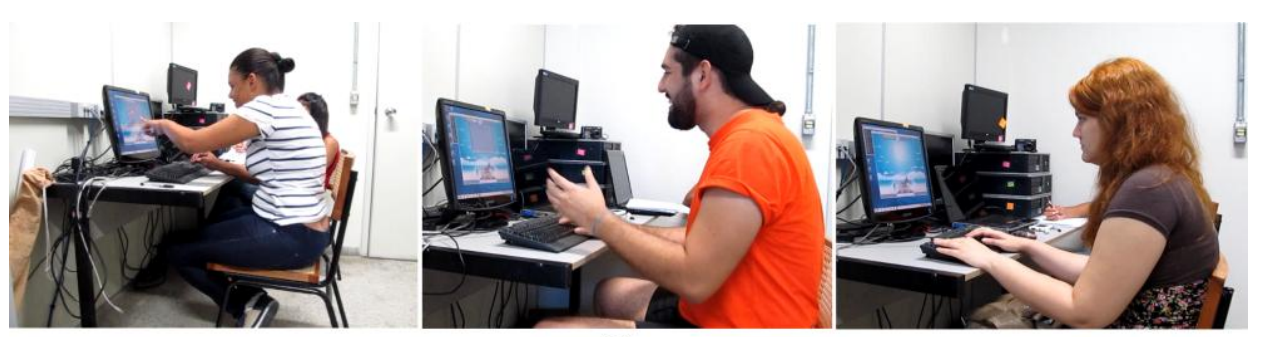

(a)

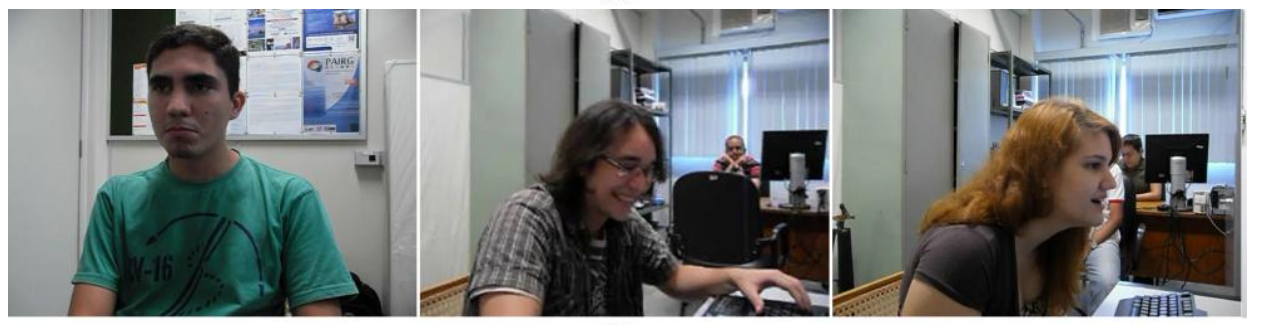

(b)

Fig. 6. Users (a) playing Karuchā Ships Invaders and (b) their reactions to the game.

\subsection{New Features}

Following, we present a list of features classified by the motivational axes, and extracted from the users' answers - optional comments and interview - in the experiment. There were 20 new features identified in this activities with student-players: Attention (FA1..FA8), Relevance (FR1..FR5), Confidence (FC1..FC3), and Satisfaction (FS1..FS4).

\footnotetext{
${ }^{9}$ All the users signed a Free and Clarified Consent Form, allowing the recording and use of their images for sake of experiment's results.
} 
- Attention: FA1. Improvement of bosses' images; FA2. Improvement of ship abduction effect; FA3. Highlight the clickable areas; FA4. Implementation of other modes, such as survival; FA5. New modes/levels with different time limit; FA6. Keep music during context-help usage; FA7. Option buttons available in the levels; FA8. More variety of sound effects.

- Relevance: FR1. Context help of previous levels on easy mode; FR2. Context help of bosses; FR3. More intuitive keyboard shortcuts; FR4. Make the most missed elements to fall with more frequency (a new mode); FR5. Implementation of a customizable mode (choose what symbols to practice).

- Confidence: FC1. Show roma-ji for missed kanas at the end of the level; FC2. Comparison of results with other players; FC3. The game give recommendations, such as "you may like/need to repeat this level" if the player misses many ships.

- Satisfaction: FS1. Implementation of a scoring system, different from number of hits; FS2. Portability to other OS; FS3. Implementation of competition/interactive mode; FS4. Development of online features (such as timeline diagrams, general stats, and achievements).

\section{Discussion}

The application of method to evaluate motivational aspects of the educational game Karuchā Ships Invaders brought to light several results from the data analysis. Analyzing the method itself, there are various questions that can be raised and discusses, such as regarding the application possibilities. In the present work, method was applied in person, and there are several characteristics of the method that can only be achieved this way, such as: usage live observations; video recording of interview; the interview itself; explanation of users' doubts by the experimenters/developers.

On the other hand, it is possible to do some adjustment in the method, in order to accomplish a future evaluation with the new online features of the game. For example, the questionnaires can be available online for the users to answer; the video for recording facial expressions can be recorded by users' webcams, if they agree to; and doubts can be solved online with FAQs or members of the evaluation team that are available to do so. Still, it is possible to consider using another evaluation method, but adapting the method to sundry situations may be important and make more sense, once it is a way to extract similar data from the in person experiment. Though, this adaptation demands more research and study.

About the game itself, there are several topics to discuss. The evaluation raised many issues that turned into new features to be implemented; which demand much work, new implementation and redesign included. The Attention axis, despite of being the one with the higher scores in the Likert-scale, was also the one who received more comments and suggestions. Several interface issues were pointed, such as the fact that some boss images contain very small or confusing representations; the ship abduction effect can make the player think he is destroying the ship instead of helping it; the clickable areas of the game are not well highlighted/indicated. These are features that will be redesigned in a next step of the research, in which the game will be completely rethought in terms of design and implementation; this is necessary to maintain the consistence of the game as a whole, once it intends to offer an immersive environment to Japanese language students, 
that would not be complete with a very well designed interface. Other new features are already solved, as keeping the music playing during context-help, more option buttons during the levels, and the new modes and levels are in design process.

The Relevance axis raised considerable questions too, as the implementation of context-help for bosses, and the possibility to navigate through the helps of previous levels. These features are already implemented, as well as another help, with less hints, for medium difficulty mode, in order to reinforce the learning experience. The new modes proposed for this axis' analysis are also in design process, as the modification of some keyboard shortcuts that the users pointed as non-intuitive.

The axis of Confidence brought few, but important new features, such as the already implemented exhibition of roma-ji for missed kanas at the end of each level. Another interesting suggestion was the idea of the game to give recommendations to the student-player, this can also reinforce the learning nature of the game. Some users also commented the desire of having their results shared and compared with other players, and this is interesting because in the interview form, a question was directed to the possibility of having an online server with some social features, though this was not implemented at all, but it was already an idea discussed by the Karuchā's development team.

The Satisfaction axis is the one whose new features are all left to implement; this axis raised more complex questions, as the development of an elaborated scoring system, the development of the game to another OS and platforms (the latter requires several thinking, once it can embrace other devices with different input characteristics), and the generation and presentation in real time of all the data, similar to what were collected in the experiment; but in the online feature, each player should have an account that saves, processes and presents all the data in graphics, diagrams and with customizable options, in addition to the option to share it to other social networks. This axis showed that there is a series of features that may seem basic and simple, but that demand complex thinking to maintain everything working and making sense, mainly in the context of an educational game, to continue offering a good balance between learning and fun, keeping also the learning context presented in the interface elements and interaction.

\section{Conclusion}

This paper presented the application of the method for evaluation of motivational aspects of educational games, describing all the steps for one specific evaluation scenario, including results. The method was applied in Karuchā Ships Invaders educational game and the results showed that the game is a powerful influence to Japanese language students, since the beginners and extending to advanced students. The evaluation also brought to light several issues that were turned into new features to be included in the game. It is noteworthy that several of these new features are already implemented after the accomplishment of the experiment.

It is important to highlight that out intention was to verify the method and the game in the motivational context. To evaluate the learning impact, it would be necessary a longitudinal study that would take months to collect data and evaluate to reach conclusions about the learning results. Which we indicate as a future work. Also, as future work, we indicate the accomplishment of a study to evaluate the possibility of 
using the method away (not in person), to achieve new data from the players of the online server, and also to verify the possibility of the method to embrace evaluation of online educational games. Also, for Karuchā, we indicate the implementation of the new features that are still open, and to introduce a katakana mode in the game.

\section{Acknowledgements}

This work was partially supported by the Brazilian National Council of Scientific and Technological Development (CNPq grant \#163408/2012-2), by the Brazilian Federal Agency for Support and Evaluation of Graduate Education (CAPES), and by the Physical Artifacts of Interaction Research Group (PAIRG ${ }^{\circledR}$ ) at Federal University of Rio Grande do Norte (UFRN), Brazil.

\section{References}

Huang, D.W., Diefes-Dux, H., Imbrie, P.K., Daku, B., \& Kallimani, J.G.: Learning motivation evaluation for a computer-based instructional tutorial using ARCS model of motivational design. In: 34th Annual Frontiers in Education, pp. 30-36 (2004)

Keller, J.: Strategies for stimulate the motivation to learn. In: Performance \& Instruction, pp. 1-7 (1987)

Marciano, J.N., Miranda, L.C., \& Miranda, E.E.C.: Aprendizagem da língua japonesa apoiada por ferramentas computacionais: estado da arte e desafios para a comunidade latino-americana. In: Proceedings of the XXXVIII Latin America Conference on Informatics, pp. 1-10. IEEE, Los Alamitos (2012)

Marciano, J.N., Miranda, L.C., \& Miranda, E.E.C.: Japanese CALL web tools: identifying and evaluating their multimedia behaviour in android OS. In: 25th World Conference on Educational Media and Technology, pp. 1087-1096. AACE, Chesapeake, USA (2013a)

Marciano, J.N., Miranda, L.C., Miranda, E.E.C., \& Pereira, R.: Android MALL apps for Japanese language: identifying and eliciting interface/interaction requirements through the semiotic framework. In: XXXIX Conferencia Latinoamericana en Informática, pp. 220-230. IEEE, Los Alamitos (2013b)

Marciano, J.N., Ferreira, A.L.S., Correia, A.C.C., Miranda, L.C., \& Miranda, E.E.C.: Karuchā Ships Invaders: cultural issues on the design/development of a Japanese CALL game made by/to Brazilians. In: XII Simpósio Brasileiro de Jogos e Entretenimento Digital, pp. 172-180. SBC, Porto Alegre, RS (2013c)

Marciano, J.N., Miranda, L.C., \& Miranda, E.E.C.: Evaluation of motivational aspects in educational media and technology. In: 26th World Conference on Educational Media and Technology, pp. 780-787 (2014)

Shellnut, B., Knowlton, A., \& Savage, T.: Applying the ARCS model to the design and development of computer-based modules for manufacturing engineering courses. In: Educational Technology Research \& Development, pp. 100-110 (1999)

Wongwiwatthananukit, S. \& Popovich, N.G.: Applying the ARCS model of motivational design to pharmaceutical education. In: American Journal of Pharmaceutical Education, pp. 188-196 (2000) 